\title{
A Low-Cost Directional Log Periodic Log Spiral Antenna
}

\author{
Emily McMilin ${ }^{1}$, Doug Henke ${ }^{2}$, Stéphane Claude ${ }^{2}$, and Jens Bornemann ${ }^{1}$ \\ ${ }^{1}$ ECE Dept, University of Victoria, Victoria, BC, V8W 3P6 \\ ${ }^{2}$ Herzberg Institute for Astrophysics, Victoria, BC, V9E 2E7 \\ E-mail: ebm@uvic.ca
}

\section{Introduction}

The Square Kilometer Array (SKA) derives its name from its total aperture area, predominately achieved by thousands of parabolic dishes, each with an antenna feed situated at its focal point. The type of antenna feed selected for SKA operation in the $1 \mathrm{GHz}$ to $10 \mathrm{GHz}$ frequency range, referred to as a Wide Band Single Pixel Feed $(\mathrm{WB}+\mathrm{SPF})[1]$, has drawn increasing attention. This paper introduces a new $\mathrm{WB}+\mathrm{SPF}$ to meet SKA scientific demands while also providing a cost-effective solution. The wide bandwidth of this new antenna is achieved by employing frequency independent (FI) design principles for the development of a log periodic (LP) antenna [2]. A basic adherence to FI principles can be seen in several of the antennas currently proposed as $\mathrm{WB}+\mathrm{SPF}$ solutions for the SKA, including the Allen Telescope Array (ATA) feed [3], the Quasi-Self-Complementary (QSC) feed [4], and the Eleven feed [5]. These antennas efficiently illuminate the radio dish with a single main lobe, ruling out entirely planar FI antennas, which generate two radiation lobes orthogonal to the plane of the antenna. High sensitivity requirements prohibit the $3 \mathrm{~dB}$ power loss that would result from attenuating one of the lobes with an absorption cavity, as is often done for directional FI antennas. Scaling the LP elements into three-dimensional space, potentially over a ground plane [6], is the present method used for directional LP antennas. We instead propose confining these often complex LP elements into a single plane, while the ground "plane" takes on a three dimensional form. Planar LP patterns permit low-cost implementations, including $\mathrm{PCB}$ and water jet cutting technology, without requiring the introduction of a complicated scaffolding to support the LP elements in 3-D. Additionally, various technologies such as metal spinning, can cost-effectively shape the ground "plane" into a 3-D structure. This low-cost WB+SPF solution would scale well in the implementation of the thousands of antenna feeds that the SKA demands. We also introduce a previously unreported LP design: the log periodic log spiral (LPLS) antenna.

\section{Design Details}

Fig. 1 shows a four-arm LPLS antenna above the ground surface modeled in CST's Microwave Studio and a prototype mounted for measurement. The zig-zag elements of Petal $_{1}$ and Petal $_{4}$ progress vertically, parallel to the $y$-axis, and the zig-zag elements of $\mathrm{Petal}_{2}$ and $\mathrm{Petal}_{3}$ progress horizontally, parallel to the $x$-axis.

$\mathrm{Petal}_{1}$ through $\mathrm{Petal}_{4}$ are attached to ports 1 through 4, respectively. The antenna operates in a differential mode between Petal $_{1}$ and Petal 4 and between Petal $_{2}$ and Petal $_{3}$. 


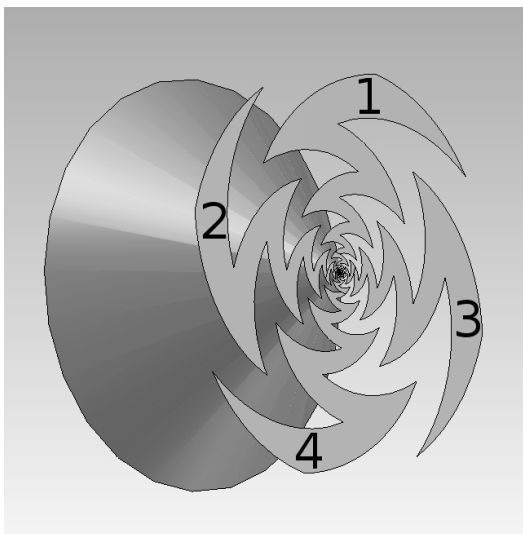

(a) CAD model

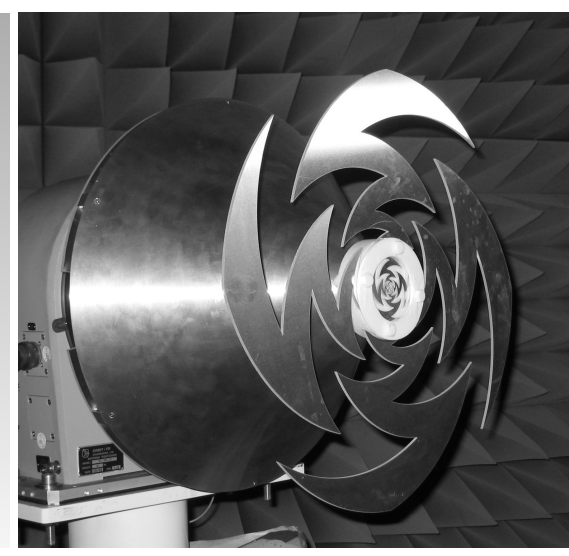

(b) Prototype

Figure 1:

a) CAD model with petals labeled and b) the prototype.

Each "zig" or "zag" of a single petal can be considered a roughly half-wave resonant element extending orthogonally from the direction that the petal progresses. These dipole-like elements radiate in a manner similar to a collinear array of dipoles, such that the E-field component of a plane wave excitation received at ports 1 and 4 is predominately horizontally polarized or parallel to the $x-z$ plane, while the E-field component received at ports 2 and 3 is predominately vertically polarized or parallel to the $y-z$ plane, achieving two linear orthogonal polarizations.

The angular extent of the zig-zags are confined within $\alpha$. In polar coordinates, the equation for one side of the $n$th "zig" or "zag" of a single petal is the basic equation for a logarithmic spiral, with some constant rate of decay, $c$, and bound by $\alpha$

$$
\rho=\left.\rho_{(n-1)} e^{ \pm c \phi}\right|_{\mp \frac{\alpha}{2}} ^{ \pm \frac{\alpha}{2}}
$$

where even values of $n$ define a "zig" using the upper + or - signs, and odd values of $n$ define a "zag", using the lower + or - signs. The extreme points at the edges of a given "zig" or "zag" are defined as $\rho_{(n-1)}$ and $\rho_{n}$ and are related to one another by the geometric scaling factor, $\tau=\frac{\rho_{n}}{\rho_{(n-1)}}<1$, by convention [2], and related to $c$ by $\tau=e^{c \alpha}$. The other side of the petal can be obtained by rotating (1) by $\phi=\frac{\pi}{i}$ radians for a total of $i$ petals.

A resonant element of length, $l$, placed a certain height, $h$, parallel to a ground plane (GP), induces an "image" current source with an opposite transverse component, an equal distance below the GP. For LP antennas, as $l$ changes, the value of $h$ must scale with the same geometric progression that produces the logarithmic growth of the resonant LP elements. Constructive interference of the radiated fields from the source and its image will be found at a height of

$$
h_{n}=k_{1} l_{n}
$$

where $k_{1}$ is a constant in the general case, and for example, equals $\frac{1}{2}$ or a quarterwavelength in the case that the element of length $l_{n}$ is a half-wave dipole [7]. The 
function that describes the shape of the ground "surface" was determined by finding the arc length of (1) from $\frac{\alpha}{2}$ to $-\frac{\alpha}{2}$ for the $n$th element

$$
l_{n}=\int_{-\frac{\alpha}{2}}^{\frac{\alpha}{2}} \sqrt{\rho^{2}+\frac{d \rho}{d \phi}} d \phi=\sqrt{1+c^{2}} \frac{\rho_{(n-1)}}{c} 2 \sinh \left(\frac{c \alpha}{2}\right)=k_{2} \rho_{(n-1)}
$$

for some constant $k_{2}$. Combining (2) and (3) with the definition of $\tau$ we find a linear relationship between the height, $h_{n}$, and the radial distance, $\rho_{n}$, for the $n$th element, specifically, $h_{n}=k_{1} k_{2} \frac{\rho_{n}}{\tau}$, determining that a conical surface is the appropriate shape for the ground surface beneath the petals.

\section{Measured Results}

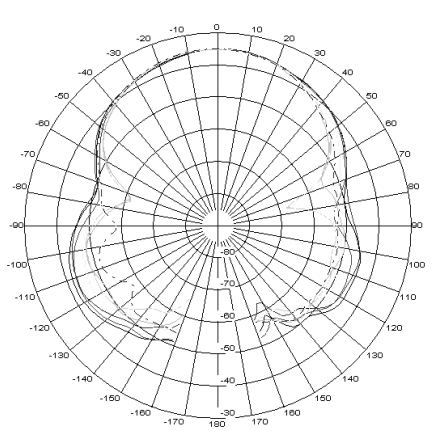

(a) $1.45 \mathrm{GHz}$

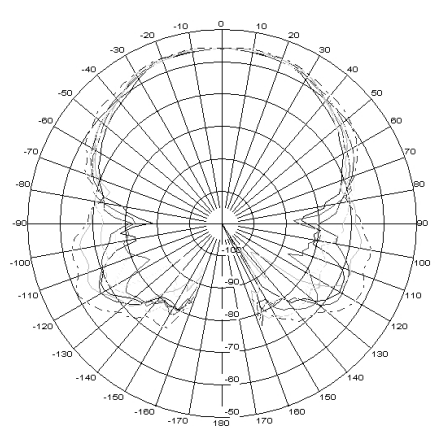

(b) $4.9 \mathrm{GHz}$

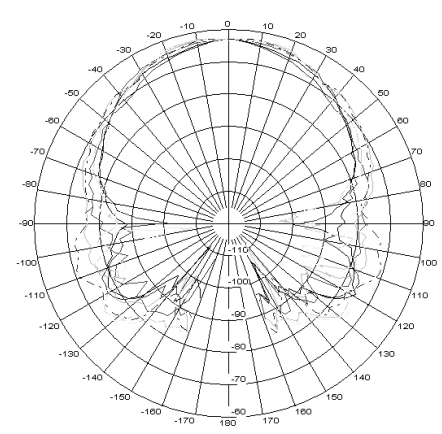

(c) $7.05 \mathrm{GHz}$

Figure 2: Co-pol radiated from (a) Petal 1 and Petal 4 , and (b), (c) Petal 2 and Petal 3 as a function of $\theta$, at $\phi=0,15,30,45,60,75$ and 90 degrees with $10 \mathrm{~dB} /$ div.

Fig. 2 shows the co-polarized E-field as a function of $\theta$ for $\theta<|160|$ degrees for the differential excitation of Petal $_{1}$ and Petal $_{4}$ (Fig. 2a), or Petal 2 and Petal 3 (Fig. 2b and Fig. 2c) at 2-D slices for multiple $\phi$ values. The polar plots show that the beam remains consistent across this range of $\phi$ values for $\theta<|40|$, the side lobe level is generally under $-10 \mathrm{~dB}$, and the $10 \mathrm{~dB}$ taper predominately occurs at the value of $\theta=|60|$ degrees. This $10 \mathrm{~dB}$ taper was designed to be comparable to that of about 65 degrees for the QSC feed [4] and about 60 degrees for the Eleven feed [5], so that similar f/D dish geometries could be used for all three feeds.

During measurement, it was found that the differential input impedance had an unexpected inductive component. This was attributed to a slight difference between the simulated CAD model and the assembled prototype, specifically, an accidental $1.5 \mathrm{~mm}$ extension of the co-axial cable outer conductor beyond

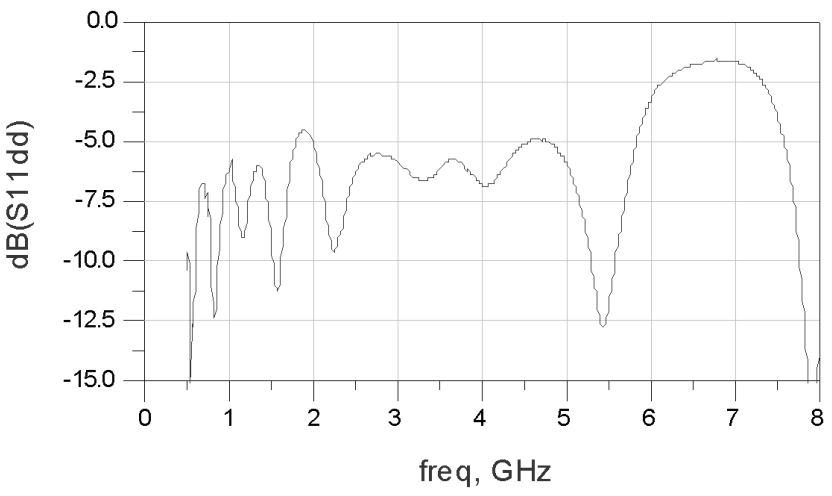

Figure 3: Measured differential input impedance, $s_{d d 11}$, after renormalization and shunt capacitor added. 
the truncated top of the cone. In the prototype, the co-axial cable outer conductor is cut flush with the cone top. To approximate this effect on the measured results, a shunt capacitance of $400 \mathrm{fF}$ was added to the input of each petal and the measured data was renormalized from $50 \Omega$ to $80 \Omega$ using a Agilent's ADS circuit simulator [8]. The differential input reflection coefficient between single-ended ports 1 and 4 shown in Fig. 3, is computed as [9],

$$
s_{d d 11}=\frac{1}{2}\left(s_{11}+s_{44}-s_{41}-s_{14}\right)
$$

as was done by both the QSC feed [10] and the Eleven feed [11]. Although the magnitude of return loss is quite poor as compared to more traditional antennas, these early results are within the realm of the early results reported for $\mathrm{WB}+\mathrm{SPF}$ antennas currently proposed for the SKA, such as the Eleven antenna [5].

\section{Conclusion}

The log periodic log spiral antenna presented in this paper provides a viable option as a $\mathrm{WB}+\mathrm{SPF}$ solution for the SKA. Measured results performed with the prototype are promising and compare reasonably well to similar antenna feeds published in the literature. One advantage of this LPLS antenna is its cost-effectiveness.

\section{References}

[1] P. Dewdney, P. Hall, R. Schilizzi, and T. Lazio, "The Square Kilometre Array," Proceedings of the IEEE, vol. 97, pp. 1482-1496, Aug. 2009.

[2] V. H. Rumsey, Frequency Independent Antennas. New York: Academic Press, 1966.

[3] J. Welch and M. Fleming, "Planned upgrade of the ATA feed," Tech. Rep. 5, US SKA Technology Development Project, March 2009.

[4] G. Cortes-Medellin, "Novel non planar ultra wide band Quasi Self-Complementary antenna," in Antennas and Propagation Society International Symposium, 2007 IEEE, pp. 5733-5736, June 2007.

[5] R. Olsson, P.-S. Kildal, and S. Weinreb, "The Eleven antenna: a compact low-profile decade bandwidth dual polarized feed for reflector antennas," Antennas and Propagation, IEEE Transactions on, vol. 54, pp. 368-375, Feb. 2006.

[6] R. DuHamel and D. Berry, "A new concept in high frequency antenna design," in IRE International Convention Record, vol. 7, pp. 42-50, Mar 1959.

[7] J. D. Kraus, Antennas. New York: McGraw-Hill, 2nd ed., 1988.

[8] Agilent Technologies, Using Circuit Simulators, May 2007.

[9] W. Fan, A. Lu, L. Wai, and B. Lok, "Mixed-mode s-parameter characterization of differential structures," in Electronics Packaging Technology, 2003 5th Conference (EPTC 2003), pp. 533-537, Dec. 2003.

[10] G. Cortes-Medellin, "Input impedance characterization of the QSC ultra wide band feed," in Antennas and Propagation Society International Symposium, 2008. AP-S 2008. IEEE, pp. 1-4, July 2008.

[11] J. Yang, X. Chen, N. Wadefalk, and P.-S. Kildal, "Design and realization of a linearly polarized Eleven feed for 1 to $10 \mathrm{ghz}$," Antennas and Wireless Propagation Letters, IEEE, vol. 8, pp. 64-68, 2009. 